\title{
Multivision: \\ An Intractable Impartial Game With a Linear Winning Strategy
}

\author{
Aviezri S. Fraenkel
}

1. Introduction. Something is definitely wrong. If the game has a linear winning strategy, then it is tractable (see Section 3). What's going on? We shall set the record straight in just a little while.

Multivision is played by two players who move alternately on a finite number of piles of finitely many tokens. A move consists of selecting a nonempty pile and changing the number of tokens in it. If the change is just any reduction in the number of tokens, we have the well-known game of Nim [1], [2]. In the present case, however, it is also permissible, normally, to increase the size of a pile by an arbitrarily large factor.

Specifically, in a multivision game $\Gamma$ we are given $m$ piles of tokens, of sizes $n_{1}, \ldots, n_{m}$. Let $M=M_{\Gamma}=\prod_{i=1}^{m} n_{i}$. Let $S=\left\{p_{1}=2, p_{2}=3, p_{3}=\right.$ $5, \ldots\}$ denote the sequence of primes. If $M>1$, let $p_{j} \in S$ be the smallest prime dividing $M$, and $p_{j+k}$ the largest. Thus $p_{j}<\cdots<p_{j+k}(k>0)$, and $\Gamma=\Gamma_{j, k}$ depends on $j$ and $k$.

A move consists of selecting any pile $i$ of size greater than 1 , and dividing its size $n_{i}$ by $p_{h}$ for some prime factor $p_{h}$ of $n_{i}$, and multiplying $n_{i}$ by $\prod_{i=h+1}^{j+k} p_{i}^{t_{i}}(\geq 1)$, where the $t_{i}$ are arbitrary nonnegative integers. Thus a move consists of reducing by 1 the exponent of some prime $p_{h}$, and increasing the exponents of all the primes greater than $p_{h}$ by arbitrary nonnegative integers. Note that if an exponent of a prime $p_{h}\left(p_{j}<p_{h} \leq p_{j+k}\right)$ was 0 in the initial position or became 0 during play, it may (again) become positive, subject to the move rule.

Play terminates when a position $u$ is reached with all pile sizes 1 , so no further move is possible. Then $M_{u}=1$. The player reaching this position wins; the opponent loses. Since multiplication by a factor at least 1 and division by a prime is involved in each move, the game is called multivision.

Note that we could have defined the game by additions and subtractions of tuples of nonnegative integers (exponents) rather than multiplications and 
divisions of products of prime powers. We would then have had to define the moves on vectors, which is less natural than piles.

Example 1. Suppose $n_{1}=\prod_{i=1}^{100} p_{i}^{p_{i}}, n_{2}=\prod_{i=1}^{100} p_{i}^{2 p_{i}}, n_{3}=\prod_{i=1}^{100} p_{i}^{3 p_{i}}$ $\left(p_{i} \in S, p_{100}=541\right)$. We make a few random initial moves. Player I selects $n_{1}$, divides $p_{50}^{p_{50}}$ by $p_{50}(=229)$ and replaces $n_{1}$ by say, $n_{1}^{\prime}=\prod_{i=1}^{49} p_{i}^{p_{i}} p_{50}^{p_{50}-1} \prod_{i=51}^{100} p_{i}^{p_{i} !}$. Now player II selects $n_{3}$, replacing $\prod_{i=1}^{100} p_{i}^{3 p_{i}}$ by say, $\prod_{i=1}^{59} p_{i}^{3 p_{i}} p_{60}^{3 p_{60}-1} \prod_{i=61}^{100} p_{i}^{T\left(3 p_{i}\right)}$ $\left(p_{60}=281\right)$, where $T(a)$ is the tower function, defined by

$$
\left.T(a)=a^{a^{a a^{a^{a}}}}\right\} a
$$

Then player I selects $n_{1}^{\prime}=\prod_{i=1}^{100} p_{i}^{s_{i}}$, replacing it by say, $\prod_{i=1}^{36} p_{i}^{s_{i}} p_{37}^{s_{37}-1} \prod_{i=38}^{100} p_{i}^{T^{\left(p_{i}\right)}\left(p_{i}\right)}$, and so on, where $T^{(a)}$ is the $a$-th iterate of the tower function $T$.

This example shows that play can continue for quite a while before it terminates - if it does terminate!

The questions we are interested in:

1. Does every play of every multivision game terminate, or are there plays that continue forever?

2. What's a winning strategy for the game if and when it exists?

3. What's the complexity of such a winning strategy? That is, how difficult is it to: (i) discover a winning strategy, (ii) compute a winning move, and (iii) consummate a winning strategy, i.e., actually reach the end of play by winning the game?

We begin by establishing a small framework.

\section{Definitions and Notations.}

- A follower (or option) of a game position $u$ is any position that is reachable from $u$ in a single move.

- The set of all followers of position $u$ is denoted by $F(u)$.

- A game position $u$ is an $N$-position if the Next player can force a win, i.e., the player moving from $u$.

- A game position $u$ is a P-position if the Previous player can force a win, i.e., the opponent of the player moving from $u$.

- The set of all $N$-positions of a game is denoted by $\mathcal{N}$ and the set of all $P$-positions by $\mathcal{P}$.

Evidently, the goal of each player is always to move from an $N$-position into a $P$-position.

The main purpose of this note is to prove 
Theorem 1. Every play of every multivision game $\Gamma$ terminates. Play at any position $u$ of $\Gamma$ can be prolonged arbitrarily long precisely as long as $M_{u}$ has a prime factor $\neq p_{j+k}$, where $M_{u}=\prod_{i=j}^{j+k} p_{i}^{s_{i}}$.

In other words, every play of $\Gamma$ terminates, but it can be prolonged arbitrarily long precisely while $M_{\Gamma}$ is divisible by a prime less than $p_{j+k}$.

Theorem 2. Every multivision game has a winning strategy for precisely one of the two players. The P-positions are precisely the set of positions $u$ for which $M_{u}$ is a square.

Note that the initial position of the game in Example 1 is a $P$-position.

Summarizing, the 2-player game multivision has the following properties:

(i) precisely one of the two players can consummate a win in a finite number of moves,

(ii) at each stage the winner can compute a next winning move in linear time,

(iii) at each step, except the last few, play can be delayed indefinitely, and

(iv) one can compute in linear time who of the two players can win and who loses, assuming the winner plays correctly (which can easily be done by (ii)).

So the conclusion is that the game does have a definite winner, that is, a player who can force a win in a finite number of moves. Moreover, the winner's winning moves can be computed linearly and we can determine the winner in linear time. Yet the game is intractable, since (iii) implies that the winner would have to live indefinitely to consummate a win. Thus the intractability part of the title is the truth, and the linear strategy is a lie, since only parts of the strategy are linear. A strategy is tractable only if all its parts are (see Section 3).

A 2-player game $\Gamma$ is impartial if, for every position of $\Gamma$, both players have the same options. Otherwise $\Gamma$ is partizan [1]. Thus Nim and multivision are impartial, and chess and Go are partizan. Impartial games are usually simpler than partizan games. Yet even the former may exhibit pathological behavior, such as demonstrated here.

\section{Proofs.}

Proof of Theorem 1. With any position $u$ of the game associate a $(k+1)$ tuple of nonnegative integers $\left(t_{j}, \ldots, t_{j+k}\right)$, which are the exponents of $M_{u}=$ $\prod_{i=j}^{j+k} p_{i}^{t_{i}}, t_{i} \geq 0$; also say that $M_{u}$ or $\left(t_{j}, \ldots, t_{j+k}\right)$ encodes the position $u$. Thus the set of all game positions corresponds to a subset $K$ of all $(k+1)$ tuples of nonnegative integers. 
Consider the lexicographic ordering $\prec$ of $K$ :

$$
\left(r_{j}, \ldots, r_{j+k}\right) \prec\left(t_{j}, \ldots, t_{j+k}\right)
$$

if there exists $h \in\{j, \ldots, j+k\}$ such that $r_{i}=t_{i}$ for all $i<h$, and $r_{h}<$ $t_{h}$. Suppose that $\left(t_{j}, \ldots, t_{j+k}\right)$ encodes position $u$. Any follower $v$ of $u$ is obtained by reducing by 1 the exponent of some $p_{h}$ in some $n_{i}$, and possibly increasing the exponents of some of the primes $>p_{h}$. Therefore $v$ is encoded by $\left(t_{j}, \ldots t_{h-1}, t_{h}-1, r_{h+1}, \ldots, r_{j+k}\right)$, where $r_{i} \geq t_{i}$ for $i \geq h+1$. Thus $v \prec u$. Since the lexicographic ordering is a well-ordering of the set $K$, play terminates after a finite number of moves.

If $u$ is a position encoded by $M_{u}=p_{j+k}^{s_{j+k}}$, then for $r>s_{j+k}$, play terminates in fewer than $r$ moves. But if $M_{u}=a p_{i}^{s_{i}} p_{j+k}^{s_{j+k}}$ where $p_{i}<p_{j+k}$, $s_{i}>0$ and all the prime factors of $a(a \geq 1)$ are less than $p_{i}$, then there exists a pile of size $a^{\prime} p_{i}^{s_{i}^{\prime}} p_{j+k}^{s_{j+k}^{\prime}}$ with $s_{i}^{\prime}>0$ and $a^{\prime}$ a factor of $a$. The move to $a^{\prime} p_{i}^{s_{i}^{\prime}-1} p_{j+k}^{s_{j+k}^{\prime}+r}$ realizes play of length $\geq r$ moves.

Proof of Theorem 2. It follows from Theorem 1 of [4] that for any game which terminates in a finite number of moves, the set of all its positions can be partitioned uniquely into subsets $\mathcal{N}$ and $\mathcal{P}$. From Theorem 3 of [4], it follows that for acyclic games, such as multivision, any partition of the game positions into $\mathcal{N}^{\prime}$ and $\mathcal{P}^{\prime}$ for which

$u \in \mathcal{N}^{\prime} \quad$ if and only if $\quad F(u) \cap \mathcal{P}^{\prime} \neq \emptyset$ and $u \in \mathcal{P}^{\prime} \quad$ if and only if $F(u) \subseteq \mathcal{N}^{\prime}$, satisfies $\mathcal{N}^{\prime}=\mathcal{N}$ and $\mathcal{P}^{\prime}=\mathcal{P}$. It thus suffices to show:

(i) Any position $u$ encoded by $M_{u}=\prod p_{i}^{s_{i}}$ such that $M_{u}$ is not a square, has a follower $v$ encoded by a square.

(ii) Every follower $v$ of a position $u$, encoded by $M_{u}=\prod p_{i}^{s_{i}}$, which is a square, is encoded by a nonsquare.

(i) If $M_{u}$ is not square, it has a smallest prime factor, say $p_{h}$, with an odd exponent $s_{h}$. Then there exists a pile of size $n_{i}$, such that $p_{h}^{s}$ divides $n_{i}, p_{h}^{s+1}$ does not divide $n_{i}$ and $s$ is odd. The move $n_{i} \rightarrow n_{i} p_{h}^{-1} \prod_{i=h+1}^{k} p_{i}^{t_{i}}$ results in a position $v$ encoded by $M_{v}$ such that $M_{v}$ is a square, if and only if $t_{i}$ has the same parity as the exponent of $p_{i}$ in $M_{u}$.

(ii) Any move $n_{i} \rightarrow n_{i} p_{h}^{-1} \prod_{i=h+1}^{k} p_{i}^{t_{i}}$, from a position encoded by a square, results in a position $v$ encoded by a nonsquare $M_{v}$, since the exponent of $p_{h}$ in $M_{v}$ is odd.

Theorem 1 states, roughly, that play can be prolonged arbitrarily long except during the "end moves". Delaying the end of play is normally in the 
interest of the loser. Nevertheless, we show explicitly that either player can effect a delay.

Corollary. Play at any position u of a multivision game $\Gamma$ can be prolonged indefinitely by either player, precisely while it is true that $M_{u}$ has a prime factor $<p_{j+k}$, where $M_{u}=\prod_{i=j}^{j+k} p_{i}^{s_{i}}$.

Proof. We first show that the winner can prolong play indefinitely. Let $M_{u}=a p_{i}^{s_{i}} p_{j+k}^{s_{j+k}}$, where $p_{i}<p_{j+k}, \quad s_{i}>0$ and all the prime factors of $a$ are less than $p_{i}$. Suppose first that $M_{u}$ is square. By Theorem 2, player II can force a win. In the worst case player I keeps reducing $s_{j+k}$. After it becomes 0 , player I is the first to reduce $s_{i}$ or the exponent of a prime less than $p_{i}$. Then player II responds by making the exponent of $p_{j+k}$ arbitrarily large, while at the same time restoring $M$ to be square. If $M_{u}$ is not a square, then player I can win by moving $u \rightarrow v$ with $M_{v}$ a square, so we are back in the previous case. The same argument shows that the loser can delay play indefinitely.

3. Complexity of Multivision. It is convenient to input the initial set of pile sizes $n_{i}$ in the form of their prime decompositions. During play it is convenient to maintain for each $n_{i}$ and for $M$ a $(k+1)$-vector $V_{i}$ and $V_{M}$ over $\mathbb{Z}_{2}$, where $V_{M}$ is the sum over $\mathbb{Z}_{2}$ of the $V_{i}$. If $n_{i}=\prod_{r=j}^{j+k} p_{r}^{s_{r}}$, then $V_{i}$ is the vector $\left(s_{j}, \ldots, s_{j+k}\right)$ over $\mathbb{Z}_{2}$. Thus for Example 1, the vectors have initially length $100, V_{1}$ and $V_{3}$ consist of 1 s except for the leftmost component, which is 0 in both, and $V_{2}$ and $V_{M}$ are the 0 -vector. Whenever the loser makes a move, $V_{M}$ becomes nonzero. The winner then locates the leftmost column of $V_{M}$, say $h$, which contains a 1 . There exists some $n_{i}$ such that $V_{i}$ contains a 1 in column $h$, so the winner can restore $V_{M}$ to 0 by dividing $n_{i}$ by $p_{h}$ and multiplying it by $p_{r}$ raised to an odd (even) power for all $r>h$ for which $V_{M}$ has a $1(0)$ in column $r$.

In addition to the binary vectors $V_{i}$, also their true values (over the ring of integers) have to be maintained for checking the validity of the move of the opponent and for recognizing the end of the game.

If $f$ and $g$ are functions from the nonnegative integers into the nonnegative reals, we write, as customary, $f(n)=\Theta(g(n))$ if there exist constants $c_{2} \geq c_{1}>0$ such that $c_{1} g(n) \leq f(n) \leq c_{2} g(n)$ for all large $n$. For complexity studies it's usually preferable to use the $\Theta$-notation rather than the $O$-notation, since if, say, $f(n)=O(\log n)$, then also $f(n)=O(n)$, so the $O$-notation doesn't necessarily differentiate between functions one of which is exponential in the other.

In the realm of discrete mathematics we say, roughly, that a problem is tractable if it can be solved in time (number of steps) that is a polynomial 
in the input length of the problem. Otherwise it is intractable. A game is tractable if: (i) the recognition of the $P$-positions, (ii) the computation of a winning move, and (iii) the consummation of the win are tractable. It is reasonable to define tractability of (i) and (ii) as for all other problems in discrete mathematics, namely computation in time polynomial in the input size. But it is unreasonable to do so for (iii): Nim is the prototype of a tractable game. Given a game of Nim with two piles, each of length $n$. The input length is then $\Theta(\log n)$, since we can represent $n$ in binary, say, which requires $\left\lceil\log _{2}(n+1)\right\rceil$ bits (binary digits). If player I keeps taking a single token from one of the piles, player II has to take one from the other pile in order to maintain a winning position, so the length of play is $\Theta(n)$, which is exponential in the input length. But a game with play length beyond a polynomial in an exponential is intractable. See [3, Section 7] for more information about the tractability of games.

The winning strategy for multivision described in the proof of Theorem 2 is linear, i.e., tractable with polynomial of degree 1 , and is similar to the winning strategy of Nim. Theorem 2 and its proof show that, in fact, (i) and (ii) of question (3) can be done in linear time. Yet the game is intractable, because the loser can normally prevent the winner's consummation of the well-deserved win indefinitely. Example 1 perhaps begins to suggest that play can last beyond anything suggested by the Ackermann function, which is the prototype of an extremely fast-growing function; see, e.g., [5, Section 5], [10, Section 2].

Summarizing this section, we may say that multivision is a simple example of an intractable game - which has a linear winning strategy if we disregard the length of play. There are games for which (i) of question (3) is provably hard (exponential), and others for which (i) and (iii) are easy but (ii) is either undecidable or its complexity is unknown. Multivision is a simpler game than the long Epidemiography games [5], [6], [7], which are related to the Hercules-Hydra game (reviewed in [11]), yet it can last much longer.

We end by describing a generalization $K$-multivision of multivision. Let $K$ be a fixed integer $\geq 2$. We begin to play $K$-multivision with $m$ piles of sizes $n_{1}, \ldots, n_{m}$ as in multivision. A move consists of selecting a prime $p_{h}$ and an integer $s<K$ and dividing some piles $n_{i_{1}}, \ldots, n_{i_{r}}$ by $p_{h}^{s_{1}}, \ldots, p_{h}^{s_{r}}$ with $\sum_{i=1}^{r} s_{i}=s$ (where $r \leq s$; and $n_{i_{1}}$ is divisible by $p_{h}^{s_{1}}, \ldots, n_{i_{r}}$ by $p_{h}^{s_{r}}$; $s_{i}$ positive integers). We also multiply $n_{i}$ by $\prod_{i=h+1}^{k} p_{i}^{t_{i}}(\geq 1)$, where the $t_{i}$ are arbitrary nonnegative integers, for $i \in\left\{i_{1}, \ldots, i_{r}\right\}$.

The special case $K=2$ of $K$-multivision is, of course, multivision. 
Theorem 3. Every play of every $K$-multivision game $\Gamma$ terminates. Play at any position $u$ of $\Gamma$ can be prolonged arbitrarily long, even by the winner, precisely as long as $M_{u}$ has a factor $<p_{j+k}$, where $M_{u}=\prod_{i=j}^{j+k} p_{i}^{s_{i}}$. Every $K$-multivision game has a winning strategy for precisely one of the two players. The P-positions are precisely the set of positions $u$ for which $M_{u}$ is a $K$-th power.

The proof is similar to that of Theorem 1; it follows from the following two easily verifiable observations:

(i) Any position $u$ encoded by $M_{u}=\prod p_{i}^{s_{i}}$ such that $M_{u}$ is not a $K$-th power, has a follower $v$ encoded by a $K$-th power.

(ii) Every follower $v$ of a position $u$, encoded by $M_{u}=\prod p_{i}^{s_{i}}$, such that $M_{u}$ is a $K$-th power, is encoded by an integer $M_{v}$ such that $M_{v}$ is not a $K$-th power.

Finally we remark that other pathological games are discussed, e.g., in [8], [9], and [13].

Confession. While reading the section on factoring a number $x$ via the difference of squares method, $x=y^{2}-z^{2}$, in the inspiring survey article [12] of Carl Pomerance, multivision formed before my eyes in a flash. The sad part is that I then promised myself to finish reading [12], which is still in the bottomless queue of things I promised myself to do. Instead I wrote this paper that same day and sent it, on an impulse, to some friends, which I immediately regretted. But some of my recipients, among them Thomas Ferguson, Daniel Ullman, and Herbert Wilf, sent me nice feedback, and Herb encouraged me to send it to the Monthly. So if you don't like this paper, blame me. If you do like it, thank Tom, Dan, and Herb.

\section{References}

1. E. R. Berlekamp, J. H. Conway, and R. K. Guy, Winning Ways (two volumes), Academic Press, London, 1982.

2. C. L. Bouton, Nim, a game with a complete mathematical theory, Ann. of Math. 3 (1902) 35-39.

3. A. S. Fraenkel, Scenic trails ascending from sea-level Nim to alpine chess, in: Games of No Chance, Proc. MSRI Workshop on Combinatorial Games, July, 1994, Berkeley, CA (R. J. Nowakowski, ed.), MSRI Publ. Vol. 29, Cambridge University Press, Cambridge, 1996, pp. 13-42.

4. A. S. Fraenkel, Combinatorial game theory foundations applied to digraph kernels, Electron. J. Combin. 4 (2) (1997) \#R10. http://www.combinatorics.org/Volume_4/wilftoc.h

5. A. S. Fraenkel, M. Loebl, and J. Nešetřil, Epidemiography-II. Games with a dozing yet winning player, J. Combin. Theory (Ser. A) 49 (1988) 129-144. 
6. A. S. Fraenkel and M. Lorberbom, Epidemiography with various growth functions, Discrete Appl. Math. 25 (1989) 53-71.

7. A. S. Fraenkel and J. Nešetřil, Epidemiography, Pacific J. Math. 118 (1985) 369-381.

8. J. P. Jones, Some undecidable determined games, Internat. J. Game Theory 11 (1982) 63-70.

9. J. P. Jones and A. S. Fraenkel, Complexities of winning strategies in diophantine games, J. Complexity 11 (1995) 435-455.

10. J. Nešetřil, Ramsey Theory, Ch. 25 in: Handbook of Combinatorics (R. L. Graham, M. Grötschel, and L. Lovász, eds.), Elsevier, Amsterdam, 1995.

11. J. Nešetřil and R. Thomas, Well quasi ordering, long games and combinatorial study of undecidability, Contemp. Math. Proc. Symp. AMS 65 (1987) 281-293.

12. C. Pomerance, A tale of two sieves, Notices AMS 43 (1996) 14731485.

13. M. O. Rabin, Effective computability of winning strategies, Contributions to the Theory of Games vol. 3, Ann. of Math. Stud. 39, 147-157, Princeton, 1957.

Department of Applied Mathematics and Computer Science, Weizmann Institute of Science, Rehovot 76100, Israel

fraenkel@wisdom.weizmann.ac.il 